\title{
Effects of Heat Stress on the Daily Behavior of Wenchang Chickens
}

\section{-Author(s)}
Li M'
Wu J'

Chen Z'

Ministry of Education Key Laboratory for Tropical Animal and Plant Ecology, Hainan Normal University, Haikou, 571158, China

\section{nail Address}

Corresponding author e-mail address Zhong Chen

Hainan Normal University, Long Kun Nan Road, Haikou City, 571158, P.R. China Email: zh.chen@hainnu.edu.cn

\section{EKeywords}

Heat stress; broiler behavior; behavioral observations; Wenchang chicken.

\section{ABSTRACT}

One-day old chicks were randomly distributed into acute heat stress (AHS) or persistent heat stress (PHS) groups. Each group was further divided into control (CK), and three AHS ages (1, 2, or 3 weeks of age) experimental subgroups. The chicks in AHS subgroups were submitted to acute heat stress $\left(40^{\circ} \mathrm{C}\right.$ for two hours between $12: 00$ and $14: 00$ hours during the weekend) and the effects of heat stress on several daily behaviors were observed. At 8 days of age, the chicks of PHS subgroups were submitted to heat stress $\left(40^{\circ} \mathrm{C}\right.$ daily). The heat treatment ceased during the weekends and the effects on the behavior were observed three times daily for three consecutive days. The results showed that, compared with the CK group, the duration and frequency of drinking and lying-down behaviors of the AHS birds increased, whereas the duration of feeding and standing significantly decreased $(p<0.01)$. The time spent walking by PHS birds was significantly longer than that of the CK groups $(p<0.01)$, and drinking was also significantly longer than that of the CK group and was significantly different when birds were three weeks old $(p=0.05)$. When heat stress lasted for two and three weeks in PHS group, the duration of lying down was longer compared with the CK group; however, this behavior was significantly shorter than the CK group when birds were three weeks old $(p<0.05)$. These results indicate that heat stress significantly affects the daily behavior of broilers, including feeding, drinking, lying, standing, and walking.

\section{INTRODUCTION}

The environmental temperature of poultry houses, in general, is not effectively controlled. Therefore, poultry production suffers huge losses due to heat stress, which is caused by high temperatures in many areas during the summer. According to a recent report, the damage caused by heat stress in poultry resulted losses of up to USD 0.728 billion in five states in the USA, including California (Carroll et al., 2012). In many parts of southern China, high environmental temperatures are frequently recorded. In particular, in Hainan Island, high temperatures occur during three quarters of the year. Therefore, poultry often suffer from heat stress, which causes inestimable and negative impacts on their growth, development, production, and reproduction (Liu and Peng, 2001).

The behavior of chickens can significantly influence their growth rate, consequently influencing production costs (Neves et al., 2010, 2014). Young chicks present high metabolism rates. While their growth rate is fast, their ability to adapt to the changes in environment conditions is poor. Moreover, since they do not present sweat glands in the skin, chickens are highly sensitive and vulnerable to heat stress, particularly when they are young. 
Heat stress has negative effects on poultry performance. For instance, Gao et al. (1999) reported that, when external house temperature was high, broiler feed intake, feed conversion ratio, and growth rate were reduced. In heat-stressed layers, reduced food intake led to a decrease in egg production and reduced egg weight and quality (Emery et al., 1984).

Heat stress can also cause changes in intestinal morphology and intestinal flora disorders (Li et al., 2015). Heat stress caused significantly reduced villus height, surface area, and volume of the small intestinal mucosa of young chicks as well as decreased the activity of digestive enzymes (Mitchell et al., 1992).

Moreover, the immune system of heat-stressed poultry may be affected. The immune organs of chicks at different ages submitted to heat stress of displayed significant atrophy and edema, necrosis of bursa cells and reduced both cell-mediated and humoral immunity (Tao et al., 1997; Tang et al., 2013). An earlier study reported that, under heat stress, chickens displayed a number of clinical symptoms, including openmouth breathing, asthma stretches, rude respiration, stretched and shrunk chest and abdomen, staggered legs, stretched body, restlessness, and weakness. The severely affected birds also presented prostration, convulsions, and even death due to heat exhaustion (Liu et al., 2007). Other studies found that under heat stress, domestic geese showed thermally induced, discontinued (interrupted) asthma, and drooping of both wings, which persisted after the heat stress period. Increased water-sticking feathers is a major behavioral response of domestic geese to resist the stimuli of high temperature (Zhong et al., 2012). These results imply that heat stress significantly affects the daily behavior of poultry and that investigating the effects of heat stress on the behaviors of the growing and developing baby chicks is of high significance. Thus, observing the effects of heat stress on different behaviors of the young chicks may reveal the characteristics and regular patterns of their response to acute heat stress (AHS) and to previous heat stress (PHS) of chicks. These findings may provide the scientific basis for correctly assessing the severity of heat stress and to establish measures to minimize the damages caused by heat stress to poultry.

\section{MATERIALS AND METHODS}

\section{Experimental birds and treatments}

One-day-old Wenchang chicks were obtained from Hainan Yongji Livestock Co. LTD (Hainan, China). Forty-eight chicks were randomly divided into an
AHS (Acute Heat Stress) group and a PHS (Persistent Heat Stress) groups. Each group was further randomly divided into a control (CK) subgroup and three ages of exposure to heat stress (1, 2, or 3 weeks of age). Heat stress consisted of submitting the birds to a temperature of $40 \pm 0.5^{\circ} \mathrm{C}$ and a relative humidity (RH) of $82 \pm 6 \%$ in an artificial climate chamber (LRH-800GS, Ming-Tian Environmental Protection Instrument Co. LTD, Shaoguan, Guangdong, China). Birds in the AHS group were submitted to HS from 12:00 to 14:00 during the weekends at 1,2 , or 3 weeks of age. In the PHS group, at 8 days of age, chicks were submitted to persistent heat stress for 1,2 or 3 weeks. The chicks in control treatment of both AHS and PHS were reared under room temperature and $\mathrm{RH}$ in artificial climate chambers at the density of $8.33 \mathrm{birds} / \mathrm{m}^{2}$.

The chicks in all groups had free access to water and feed. The broiler house was supplied with sufficient water, natural ventilation, and natural light-dark cycle, and was regularly cleaned and disinfected. The procedures of this experiment were approved by the Animal Experimentation Ethics Committee of Hainan Normal University.

\section{Behavior observational methods and recording}

A Camlife Image Recording Instruments V11.50 was acquired from TianMin Products Science and Technology Development Co., LTD (Shenzhen, Guangdong, (hina) and was used to observe and record the daily behaviors of lying down, sleeping, walking, standing, feeding, and drinking during the entire experimental period. Behaviors were recorded in AHS group during the period of heat stress $(4 \times 0.5 \mathrm{hr}=2 \mathrm{hr})$. In the PHS group, behaviors were observed and recorded at 9:009:30 h, 12:00-12:30 h, and 17:00-17:30 $\mathrm{h}$ for three consecutive days $(3 \times 0.5 \mathrm{hr}$ per day). The duration of each behavior by each individual bird was recorded using a stopwatch, and their mean weekly values were calculated.

Changes in the duration of behaviors in response to heat stress of the experimental groups were calculated.

\section{Statistical analysis}

The experimental data were analyzed using Excell2003 and means were compared by independentsample T-test (SPSS17.0 software). Data are expressed as mean \pm standard error of the mean (SEM). Differences between treatment with $p<0.05$ was regarded as statistically significant, while difference between treatments with $p<0.01$ was regarded as highly significant. 


\section{RESULTS}

\section{Effects of Heat Stress on Chicks' Lying Behavior}

The effects of heat stress on chicks' lying behavior were shown in Fig. 1. As shown in Fig. 1A, heat stress significantly affected chicks' lying behavior. The duration of this behavior was gradually reduced in both CK and AHS groups as birds aged. However, heat-stressed chicks lied down significantly longer than CK chicks. For the chicks at the same weekly age, the difference in lying-down time between the CK and the AHS groups gradually became larger. At seven days of age, the duration of lying-down sleeping of CK chicks was $18.61 \pm 0.46 \mathrm{~min}$, whereas that of the AHS chicks was $22.44 \pm 1.39 \mathrm{~min}$. At 21 days of age, the duration of lying-down of chicks in CK group and in AHS groups were $4.78 \pm 1.24$ and $14.77 \pm 4.54$ min, respectively. As shown in Fig.1B, under PHS, chicks' lying-down
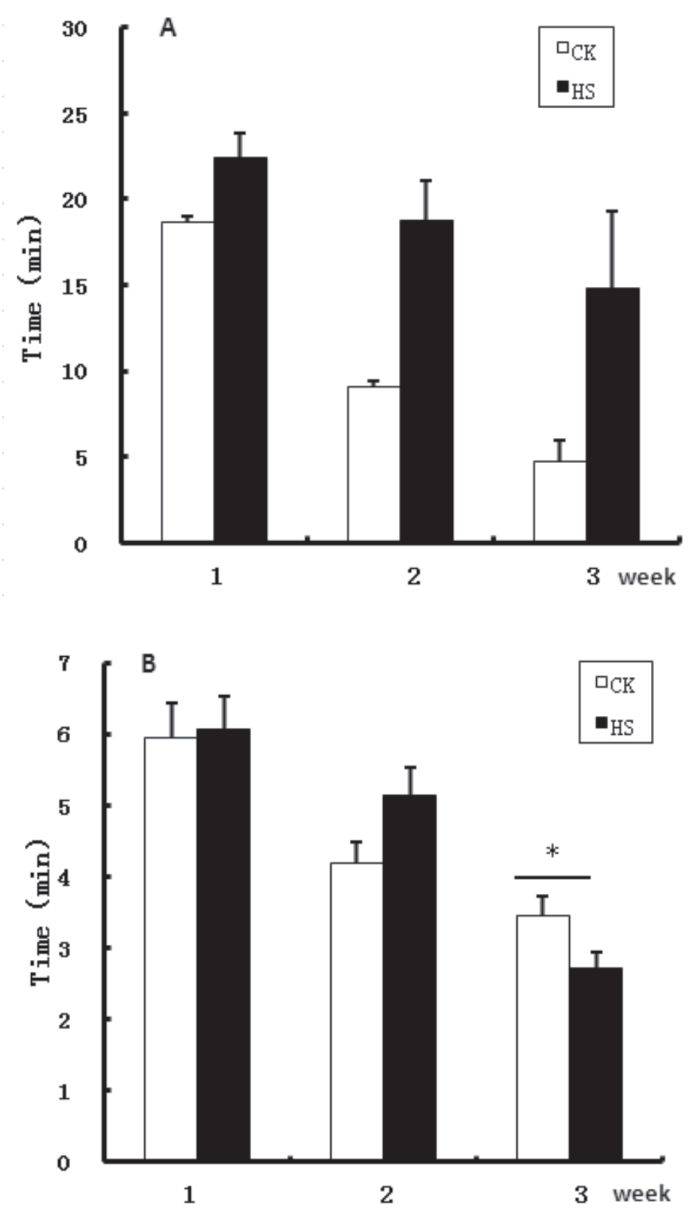

Figure 1 - Effects of heat stress on the lying behavior of chickens A. Effects of AHS on chick's lying behaviors;

B. Effects of PHS on chick's lying behavior; ${ }^{*} p<0.05 ;{ }^{* *} p<0.01, n=6$. AHS: acute heat stress; PHS: persistent heat stress. duration in both CK and experimental groups gradually decreased as birds aged. Chick's lying-down sleeping duration in the CK group was reduced from $5.95 \pm 0.49$ $\min$ to $3.46 \pm 0.27 \mathrm{~min}$, whereas in the experimental groups, the duration ranged from $6.06 \pm 0.47 \mathrm{~min}$ to $2.72 \pm 0.23 \mathrm{~min}$. When broilers were seven and 14 days old, lying-down in the PHS group experimental groups were numerically longer, but not statistically different compared with the CK group. However, at 21 days of age, lying-down duration in PHS chickens was significantly shorter than that of chicks in the CK group ( $p=0.042)$.

\section{Effects of Heat Stress on Chick Standing Behavior}

The effects of heat stress on chicks' standing behavior in response to both AHS and PHS are shown in Fig. 2, which indicates that heat stress significantly affected this behavior. Standing time of the chicks in
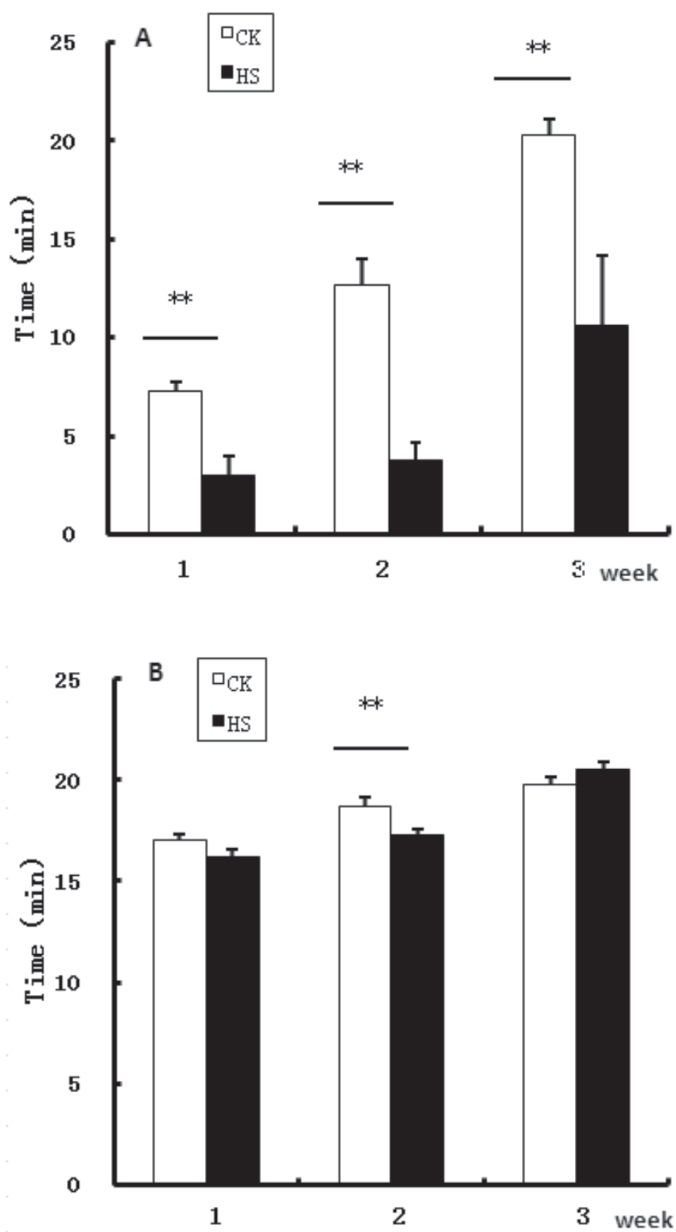

Figure 2 - Effects of heat stress on standing behaviors of chickens A. Effects of AHS on chick's standing behavior;

B. Effects of PHS on chick's standing behavior. ${ }^{*} p<0.05 ;{ }^{* *} p<0.01(n=6)$; AHS: acute heat stress; PHS: persistent heat stress. 
the CK group gradually increased from $7.28 \pm 0.43 \mathrm{~min}$ to $20.27 \pm 0.82 \mathrm{~min}$ as birds weekly age increased. At 21 days of age, the observational time for standing behavior accounted for 2/3 of total observational time. Under AHS, standing times of the experimental groups were significant shorter than that of the CK group $(p=0.000)$. As shown in Fig.2B, standing time of the CK group gradually increased with weekly age. Under PHS, standing time of the experimental groups also gradually increased with weekly age. Standing time of the CK group increased from $17.04 \pm 0.31 \mathrm{~min}$ to 19.74 $\pm 0.41 \mathrm{~min}$, whereas those of the experimental groups increased from $16.20 \pm 0.32 \mathrm{~min}$ to $20.54 \pm 0.32 \mathrm{~min}$. when broilers were 7 and 14 days old, standing times of the experimental groups were shorter than that of the CK group. However, at 21 days of age, standing time of the experimental groups was longer than that of the CK group. Only when broilers were 14 days old, standing time of the CK group (18.72 $\pm 0.38 \mathrm{~min})$ was significantly longer $(p=0.003)$ than that of the experimental groups $(17.28 \pm 0.30 \mathrm{~min})$.

\section{Effects of Heat Stress on Chick's Eating Behavior}

The effects of AHS and PHS on chicks' eating behavior are shown in Fig. 3. Fig. 3A demonstrates that AHS significantly affected eating behavior. Eating duration of CK chicks firstly increased and then decreased from 1 week to 3 weeks of age. Eating duration of AHS birds was shorter than that of the CK group, especially at 2 weeks of age $(P=0.000)$. Eating duration of AHS chickens was, on average, only $1 / 3$ of that of the CK group. As shown in Fig.3B, eating duration of the CK group gradually increased with weekly ages. Under PHS, eating duration also gradually increased with increasing weekly ages $(P=0.000)$. Eating duration of the CK group increased from $3.73 \pm 0.32 \mathrm{~min}$ to $4.39 \pm 0.31 \mathrm{~min}$, whereas in the PHS groups increased from $3.02 \pm 0.25 \mathrm{~min}$ to $4.02 \pm 0.30 \mathrm{~min}$ over the experimental period. Eating duration of PHS chickens was numerically shorter, but not statistically different, from that of CK chickens.

\section{Effects of Heat Stress on Chicks' Drinking Behavior}

The effects of heat stress on drinking behavior are presented in Fig. 4. Fig. 4A shows that drinking duration of the CK group remained almost unchanged as birds aged ( $0.085 \pm 0.003 \mathrm{~min})$, whereas under AHS, drinking duration increased from $0.143 \pm 0.022 \mathrm{~min}$ to $0.223 \pm 0.059 \mathrm{~min}$ with the increasing weekly age. Drinking duration of AHS birds were significant longer than that of the CK group when birds were 14 days old $(P=0.000)$. As shown inFig.4B, drinking duration of the CK group gradually increased from $0.023 \pm$ $0.004 \mathrm{~min}$ to $0.082 \pm 0.005 \mathrm{~min}$ with increasing weekly age. Under PHS, drinking times heat-stressed birds also gradually increased from $0.029 \pm 0.002 \mathrm{~min}$ to $0.091 \pm 0.010 \mathrm{~min}$ with the increasing weekly age and were longer than that of the CK group. However, drinking duration of PHS birds was significantly longer than that of CK group only when broilers were 21 days old $(p=0.015)$.
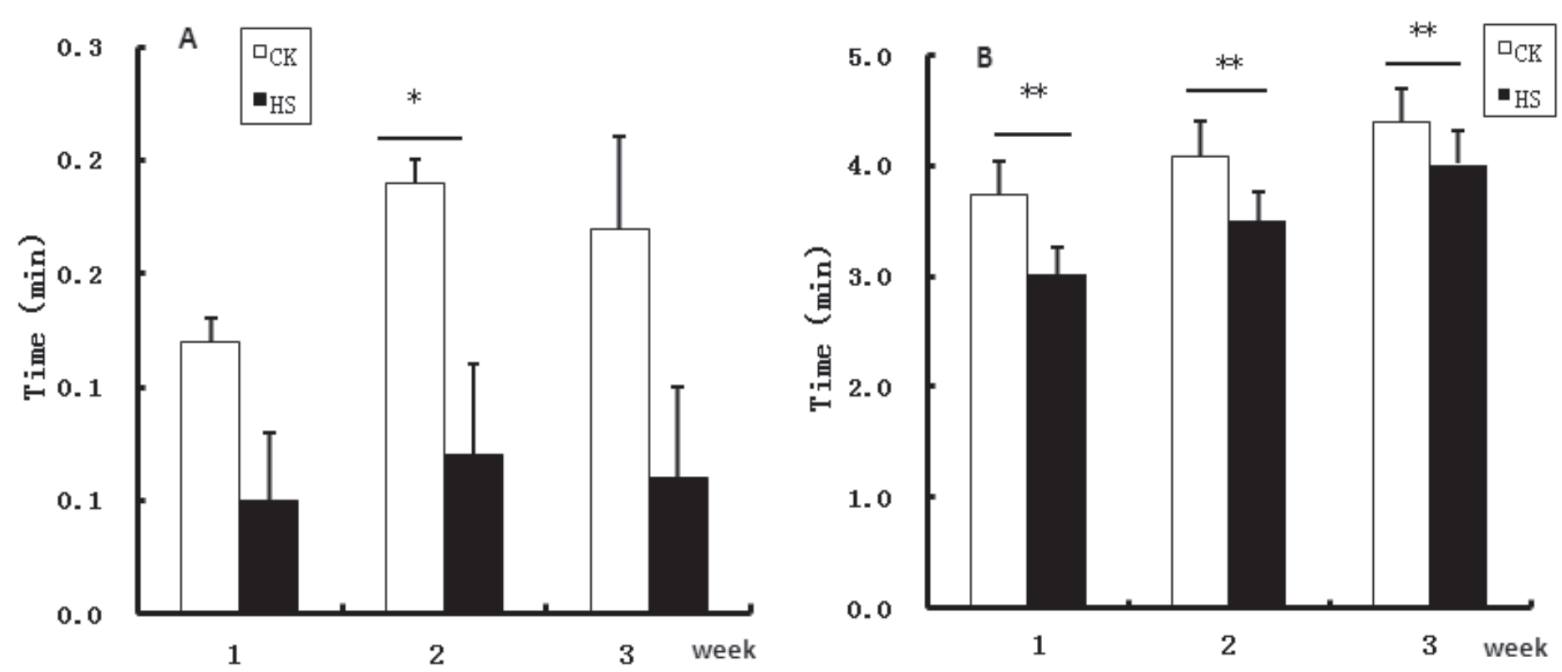

Figure 3 - Effects of heat stress on the eating behaviorsof chickens

A. Effects of AHS on chick's eating behavior;

B. Effects of PHS on chick's eating behavior. ${ }^{*} p<0.05 ;{ }^{* *} p<0.01, n=6$. AHS: acute heat stress; PHS: persistent heat stress. 

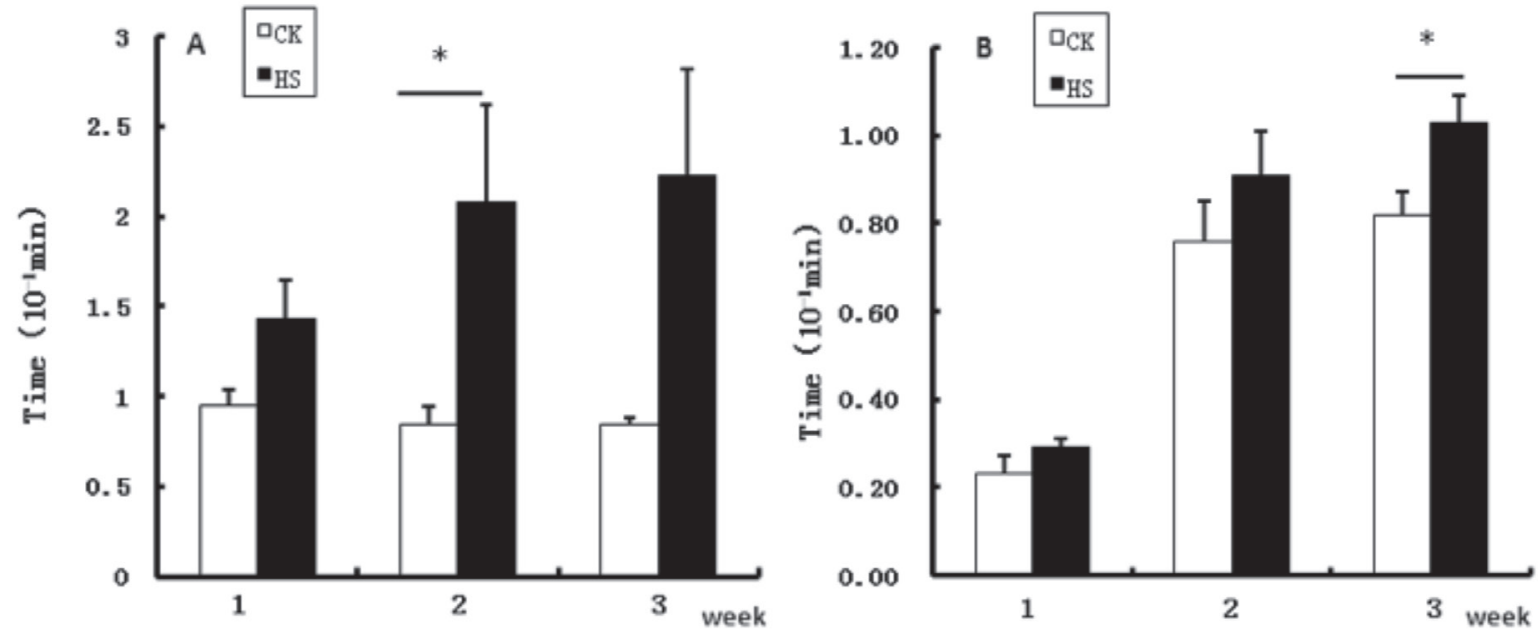

Figure 4 - Effects of heat stress on the water-drinking behaviors of chickens A. Effects of AHS on chick's drinking behavior;

B. Effects of PHS on chick's drinking behavior. ${ }^{*} p<0.05 ;{ }^{* *} p<0.01 ; n=6$. AHS: acute heat stress; PHS: persistent heat stress.

\section{Effects of Heat Stress on Chicks' Walking} Behavior

The effects of heat stress on walking behavior are presented in Fig. 5.As shown in Fig. 5A, under AHS, walking duration of one-week-old chicks of the experimental groups was longer than that of the CK group. When broilers were 14 and 21 days old, walking duration was numerically longer, but not statistically different, in the CK group compared with that of AHS birds. As shown in Fig. 5B, walking duration of CK birds displayed initially an increasing and then a decreasing trend as birds aged. Under PHS, walking duration of heat-stressed birds also displayed a similar trend of that of the CK group. However, walking duration of PHS birds was longer than that of the CK group $(p<0.01)$. At the end of the second week, walking duration of the CK group was $2.31 \pm 0.80 \mathrm{~min}$, whereas that of the PHS group was $3.29 \pm 0.10 \mathrm{~min}(p=0.000)$. At the end of third week, walking time of the CK group was $2.14 \pm 0.09 \mathrm{~min}$, whereas that of the PHS group was $2.53 \pm 0.11 \mathrm{~min}(p=0.009)$.
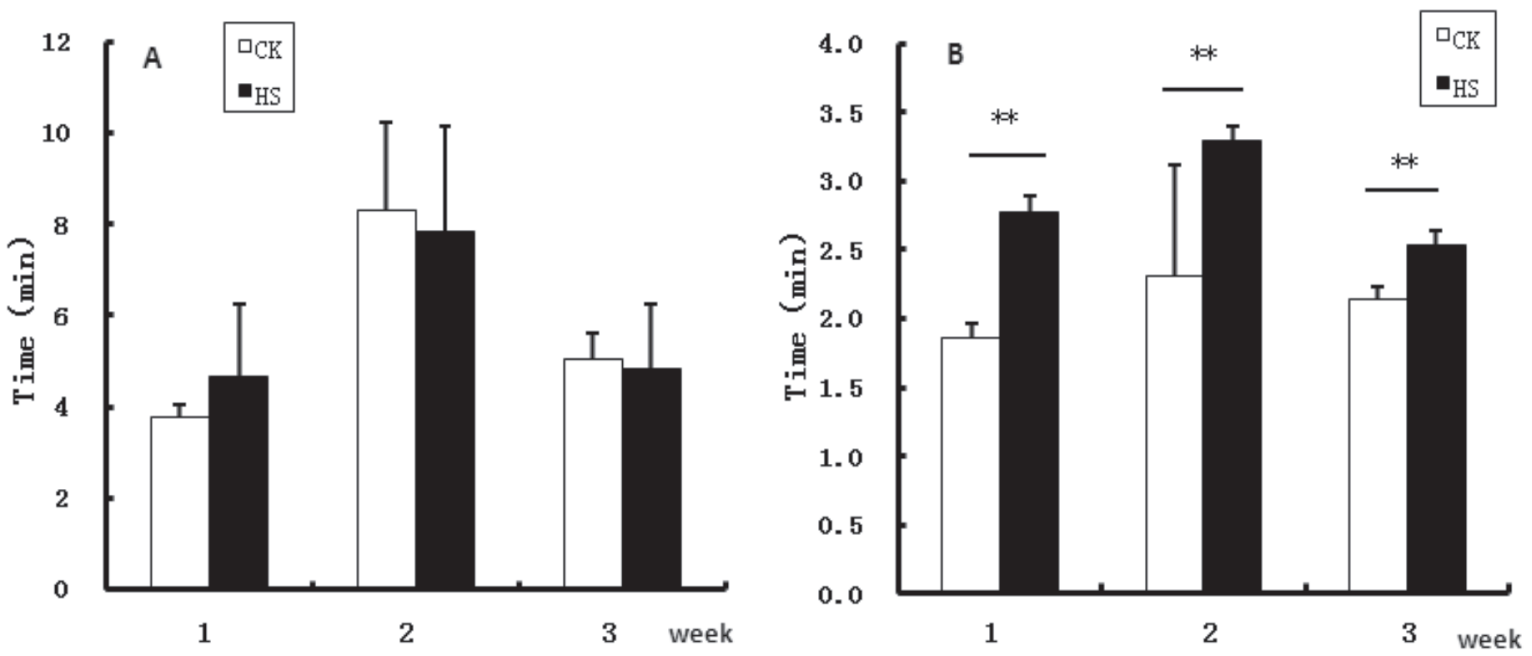

Figure 5 - Effects of heat stress on the walking behaviors of chickens A. Effects of AHS on chick's walking behavior;

B. Effects of PHS on chick's walking behavior; ${ }^{*} p<0.05 ;{ }^{*}{ }^{*} p<0.01(n=6)$ AHS: acute heat stress; PHS: persistent heat stress. 


\section{Effects of Heat Stress on Other Behaviors}

The chicks submitted to heat stress remained longer lying with their facedown on the bottom of the cage than the control birds, and it was also observed that they pecked more frequently the drinkers, feeders, and cage floor, as well as other birds, often plucking other birds' feathers (data not shown). Lying face down duration was longer of heat-stressed individuals than that of CK birds. In the afternoon, HS birds scratched the cage floor with both feet, actively flapped their wings, and continuously walked inside the cage.

\section{DISCUSSION}

In a previous study (Zhou et al., 2004), . it was observed that, under heat stress, some chicks displayed a number of clinical symptoms, including difficult breathing, open-mouth breathing, severe feed intake reduction, loss of appetite, restlessness, reduced activity and lying face-down at the bottom of the cage. In the present study, we observed that, under both acute and persistent heat stress, chicks not only displayed restless walking behavior, but also spread out their wings, laid facedown on the bottom of the cage, and panted. Birds submitted to PHS showed remained longer lying with their heads down on the bottom of the cage, pecking the drinker, the feeder, and the bottom of the cage, as well as pecking the other birds and plucking another bird's feathers than the controls. These observations are consistent with those described by previous investigators (Zhou et al., 2004). Chicks may display these abnormal behaviors to resist and/or to prevent heat stress induced by high environmental temperatures. In the present experiment, independently of heat stress being acute or persistent, heat-stressed birds presented shorter feeding and longer drinking duration compared with the control birds. These behavioral responses demonstrate that heat stress significantly affects ingestive broiler behavior.

The duration of lying down of CK birds tended to increase as birds aged, from 60 to $16.7 \%$ of the total observational time. Under AHS, lying down duration also tended to increase with bird age, but was longer compared with that of the CK group. Because the metabolism of young chicks is very active, when submitted to heat stress, chicks reduce their physical activity in an attempt to decrease body heat generation. Therefore, they lied down and slept to decrease their basal metabolic rate and to resist to heat stress.

Standing time gradually increased as birds aged. At three weeks of age, standing time of the CK group accounted for $66.7 \%$ of the total observational time, which was almost twice as high as that observed in the acute heat-stressed groups. Standing time of the acute heat-stressed groups was always shorter than that of the CK group, independently of age.

Under heat stress, eating behavior duration was also significantly altered. Eating duration of the birds submitted both to acute and persistent heat-stressed birds was significantly shorter compared with the controls. We observed that heat stress also increased drinking duration when birds were submitted either to acute or to persistent heat stress. Under heat stress, $80 \%$ of heat generated by the chick's body is dissipated by evaporation. Increasing drinking is beneficial for chick's survival, as it alleviates the effects of heat stress.

The chicks submitted to PHS frequently walked around the cage and were physically very active. Their walking duration was longer than that of the CK group. Walking time in the CK group accounted for $2 / 3$ of the total behavior observation time. In the control birds at seven, 14, and 21 days of age, drinking accounted for only a very small proportion of the total observational time, whereas the heat-stressed birds drank significantly longer than the CK group. Drinking times in both control and PHS birds tended to increase as birds aged. Drinking duration in PHS birds increased daily. This suggests that the drinking control in the central nervous system was partially inhibited by heat stress in PHS chicks and the chicks drink more water to alleviate the heat load caused by PHS. In summary, in face of PHS, chicks changed their behavior, as shown by the reduced duration of feeding and increased duration of drinking and walking.

Both AHS and PSH affected broiler behaviors to different extents. For instance, the effects of AHS on lying down, walking, drinking, and eating behaviors were significantly stronger compared with those caused by PHS. Therefore, chicks are less capable of coping with acute high temperature. The display of behaviors that avoided the heat stress caused by acute high temperature were more obvious and vigorous.

The effects of heat stress on these behaviors were also different as a function of broiler age. Older birds were less capable to cope with high temperatures as compared to young birds. For instance, under AHS, drinking times of $\mathrm{CK}$ birds with one, two and three weeks of age were $0.095 \pm 0.009 \mathrm{~min}, 0.085 \pm 0.009$ min, and $0.085 \pm 0.003$ min, respectively, whereas those submitted to heat stress presented drinking times of $0.143 \pm 0.022 \mathrm{~min}, 0.208 \pm 0.054 \mathrm{~min}$, and 


\section{Effects of Heat Stress on the Daily Behavior of Wenchang Chickens}

$0.223 \pm 0.059 \mathrm{~min}$ at the same ages, respectively. However, the drinking times of the chickens submitted to persistent heat stress were not significantly different at two and three weeks of age. Therefore, heat stress causes more serious damage to older chickens than to younger ones.

The metabolism of young chicks is very active. Hot environmental temperatures make it difficult for chickens to dissipate the heat because they do not present sweat glands on their skin. When challenged by heat stress, they reduce body heat generation by reducing their physical activity and their basal metabolism rate mainly by lying down and sleeping, as well as their feed intake. A related study indicated that exposure of broiler chicks to heat stress for two weeks reduced daily food intake by $2.67 \%$ (Li et al., 2000). Another study also observed that, within the temperature range of $22^{\circ} \mathrm{C}-32^{\circ} \mathrm{C}$, feed intake was reduced by $3.6 \%$ for every $1^{\circ} \mathrm{C}$ increase in environmental temperature (Baziz et al.,1996).It was also recently reported that, in broilers submitted to heat stress, the small intestinal wall was thinner and the small intestinal mucosal epithelial cells appeared to disperse outwards. In many intestinal segments, mucosal epithelial cells were detached, accompanied by exposed laminapropria and ruptured small intestinal villi (Chen et al., 2015). The effect of damages to the small intestine on broiler feed intake is huge. Heat stress leads to reduced feed intake in many other animals. This reduced feed intake is also related with animal age, species, and the different temperature ranges in the rearing environment (Geraert et al., 1996). Different researchers carried out studies with different animal species and under different experimental conditions, and therefore, the results obtained are somewhat different. However, broiler studies demonstrated that heat stress significantly affects their eating behavior. Submitting broilers to high temperatures caused highly significant and negative impacts on daily weight gain, feed intake, and feed to gain ratio of broilers chicks, and seriously affected their growth rate, physiological mechanisms, and health status (Li et al., 2000), consequently affecting their growth performance (An et al., 2014).

In many parts in southern China, during the long and hot summer season, broilers are submitted to heat stress for much longer than the acute and persistent heat stress periods applied in the present study. Therefore, the natural heat stress that broilers suffer during the summer in chinamay cause huge performance losses.
In general, feed intake and physical activity generates heat. This study showed that, during the heat-stress periods applied, chickens decreased eating time to reduce heat generation. Drinking duration showed the opposite trend. Zhong et al. (2012) found that in broilers submitted to heat stress, drinking was dramatically increased and the amount of feed intake was correspondingly reduced. The results of the present study indicated that both AHS and PHS cause heat stress in broilers, but that AHS caused more serious damage to the broilers than PHS. The duration of daily behaviors, such as feeding, drinking, lying down, standing, and walking, of the broilers submitted to heat stress were very different relative to the control broilers. The birds attempted to alleviate the damaging effects of heat stress by increasing the time spent drinking and reducing the time spent eating, standing times.

\section{ACKNOWLEDGEMENTS}

This study was funded by research grants from National Natural Science Foundation of China (NSFC31260555) and Science Foundation of Hainan Normal University (HSHX2011-10;CXCYXJ2013013).

\section{REFERENCES}

Ahir VB, Singh KM, Tripathi AK, Mathakiya RA, Jakhesara SJ, et al. Study of bacterial diversity in poultry gut using denaturing gradient gel electrophoresis. Iranian Journal of Applied Animal Science 2012,2(3):227-232

An LL, Yang JJ, Li ZY, Wu JW, Tang PX. Influence of lutein-traditional Chinese medicine on production performance and behavior of laying hens at high environmental temperature. Journal of Domestic Animal Ecology 2014;35(7):21-26

Baziz HA, Geraert PA, Padiha JCF, Guillaumin S. Chronic heat exposure enhances fat deposition and modifies muscle and fat partition in broiler carcass. Poultry Science 1996;75(4):505-513.

Carroll JA, Burdick NC, Chase Jr CC, Coleman SW, Spiers DE. Influence of environmental temperature on the physiological, endocrine, and immune responses in livestock exposed to a provocative immune challenge. Domestic Animal Endocrinology 2012;43(2):146.

Chen Z, Xie J, Hu MY, Tang J, Shao ZF, Li MH. Protective effects of $\nabla-$ aminobutyric acid (GABA) on the small intestinal mucosa in heatstressed Wenchang chicken. Journal of Animal and Plant Sciences 2015;25(1):78-87

Emery DA, Vohra P, Ernst RA. The effect of cyclic and constant ambient temperatures on feed consumption, eggs production, eggs weight and shell thickness of hens. Poultry Science 1984;63(10):2027-2035.

Gao ZB, Fu WL, Jiang ZY, Lin YC, Yu DQ, Wu WY. Effect of high ambient temperature on production performance and level of plasma thyroid hormone and insulin of broilers. Journal of South China Agricultural University 1998;20(1):26-31. 
Geraert PA, Padilha JCF, Guillaumin S. Metabolic and endocrine changes induced by chronic heat exposure in broiler chickens: growth performance, boby composition and energy retention. British Journal of Nutrition 1996;75(2):195-204.

Li SY, Zhang MH, Zhang ZY, Yan S., Du R, Wang KL, et al. Effect of heat stress on performance of broilers and some blood biological and endocrinological variate. Acta Agricuturae Boreali-Sinica 2000;15(3):140-144.

Li YZ, Li J, Zhang NB, Chen CX, Cui YQ. Diversity analysis of the intestinal microbial flora of laying hens underheat stress. Acta Ecologica Sinica 2015;35(5):1-13.

Liu JL, Gong XT, Sun YA, Hu HJ. Occurrence and control of heat stress of chicken. Chinese Journal of Animal Husbandry and Veterinary Medicine 2007;(11):93-94

Liu MS, Peng JS. Research advance of effects on heat stress in domestic bird. Feed Industry 2001;22(3):13-15.

Mitchell MA, Carlisle AJ. The effect of chronic exposure to elevated environmental temperature on intestinal morphology and nutrient absorption in the domestic fowl (Gallus domesticus). Comparative Biochemistry and Physiology Part A: Physiology 1992;101(1):137-142.
Neves DP, Banhazi TM, Nääs IA. Feeding behaviour of broiler chickens: a review on the biomechanical characteristics. Brazilian Journal of Poultry Science 2014;16(2):1-16

Neves DP, Nääs IA, Vercellino RA, Moura DJ. Do broilers prefer to eat from a certain type of feeder? Brazilian Journal of Poultry Science 2010;12(3):179-187.

Tang J, Chen LY, Wang B, Shao ZF, Zhang JH, Chen Z. Effects of GABAmorinda officinalis root polysaccharide compound on growth performance and immune function of chicks. Journal of Domestic Animal Ecology 2013;34(8):41-47.

Tao XP, Xia D, Li RZ. Effect of heat stress on the immune response of chicken. Chinese Poultry 1997;(6):4-6.

Zhong QZ, Wang D, Sun ZW, Lou YJ. Behavior observation of goose in the period of heat stress. Animal Production 2012;48(3):60-62.

Zhou BH, Jiang GJ, Wang P. The harm and prevention measures of heat stress of chicken. Chinese Journal of Veterinary Drug 2004;38(7):4142. 